\title{
Intestinal Protothecosis in a Young Bengal Cat
}

\author{
Sara Manfredini' ${ }^{1}$, Luca Formaggini' ${ }^{1}$, Michele Marino², Luigi Venco ${ }^{{ }^{*}}$ \\ ${ }^{1}$ Clinica Veterinaria Lago Maggiore, Dormelletto (NO), Italy \\ ${ }^{2}$ Laboratorio Analisi Veterinarie La Vallonea, Passirana di Rho (MI), Italy \\ Email: *luigivenco@libero.it
}

How to cite this paper: Manfredini, S., Formaggini, L., Marino, M. and Venco, L. (2021) Intestinal Protothecosis in a Young Bengal Cat. Open Journal of Veterinary Medicine, 11, 157-164.

https://doi.org/10.4236/ojvm.2021.115011

Received: March 19, 2021

Accepted: May 15, 2021

Published: May 18, 2021

Copyright $\odot 2021$ by author(s) and Scientific Research Publishing Inc. This work is licensed under the Creative Commons Attribution International License (CC BY 4.0).

http://creativecommons.org/licenses/by/4.0/

\begin{abstract}
Background: Intestinal protothecosis is an uncommon and insidious mycotic disease. Only one human case and a few rare cases in dogs have been reported. To the authors' knowledge, intestinal protothecosis has never been reported in cats. Case description: This paper describes a case of intestinal protothecosis in a nine-month-old male, Bengal cat. The cat presented because of onset of haemorrhagic diarrhoea. Investigations allowed diagnosis of intestinal protothecosis, confirmed by PCR test on faeces. Treatment with itraconazole did not improve the clinical signs. Treatment with nystatin was prescribed and caused improvement in the clinical signs and decreased number of pathogens seen on faecal cytology. PCR on faecal samples was negative two months after treatment, with complete resolution of symptoms. Conclusion: Infection with Prototheca should be part of the list of differential diagnoses for diarrhoea in cats. nystatin was effective in treating the infection in this case; this drug should be considered as a first-line treatment in cats as well as in dogs, in which protothecosis appears to have a poor prognosis. Although protothecosis is not considered a zoonotic disease, cases of algal infections in companion animals might be considered indicators of environmental risks for humans.
\end{abstract}

\section{Keywords}

Chronic Diarrhoea, Haemorrhagic Colitis, Feline Medicine, Intestinal Protothecosis, Prototheca Infection

\section{Introduction}

Protothecosis is an uncommon cutaneous or systemic disease caused by Prototheca spp., which are unicellular algae [1]. These algae lack chlorophyll, have a saprophytic life cycle, with a worldwide distribution except Antarctica [2]. They favour warm, humid climates where there is abundant organic matter with high water content [1]. Prototheca cells appear ovoid or oblong in tissue section and 
spherical in suspension, with diameters ranging from 1.5 to $30 \mu \mathrm{m}$, granularbasophilic cytoplasm and a thick cell wall [3] [4]. Currently recognized Prototheca species include: P. zopfii, $P$. wickerhamii, P. blaschkeae, P. stagnora, $P$. ulmea and $P$. cutis. Only $P$. zopfii and $P$. wickerhamii cause disease in dogs and cats [1]. Clinical presentations of human protothecosis include localized infections such as cutaneous or subcutaneous infections or bursitis, which occur in immunocompetent hosts and usually result from traumatic inoculation. Disseminated infections can occur in immunocompromised hosts [4]. One case of human intestinal protothecosis has been reported [5]. Fewer than 50 cases have been reported in dogs, with most relating to single cases from North America and Australia [6] [7]. Interestingly, even fewer confirmed cases have been documented in Europe, from Germany, Italy, Poland, Spain and the United Kingdom [8]. In dogs, protothecosis is usually a serious disseminated disease, but localized cutaneous disease occurs occasionally [1]. Most affected dogs do not have a history of immunosuppressive drug therapy or illness. Boxer and Collies breeds may be predisposed, possibly secondary to an underlying genetic immunodeficiency, although a variety of other small and large breed dogs also can be affected [8]. Systemic invasion probably occurs after ingestion of a large number of microorganisms and colonization of the colon and then the rectus. Haematogenic spread, especially in the setting of concurrent ulcerative disease, e.g. granulomatous colitis of Boxer dogs [2], can lead to ocular and central nervous system involvement [9]. Protothecosis is very rare in cats because of either natural resistance to infection or avoidance of environmental niches where algae are typically found [9]. The few published cases were all described in clinically healthy adult cats with firm, non-ulcerated, cutaneous or subcutaneous masses on the forehead, distal limbs, tail base, nose, or pinnae. Affected cats were typically FIV and FeLV negative were otherwise in good health and aged from 3 to 16 years [10]. The absence of regional lymphadenomegaly and the lack of any clinical signs of systemic disease in these cats suggest that the infections were localized. One cat developed new, distant nodules several months after excisional biopsy of an original solitary lesion [11]; systemic disease has not been reported.

We present a case of a cat affected by intestinal protothecosis diagnosed in North-West Italy in December 2018.

\section{Case Details}

A six-month-old Bengal male cat of $1.2 \mathrm{~kg}$ bodyweight was presented to our clinic because of acute onset of hemorrhagic large bowel diarrhoea in the previous 5 days. The cat was one of five; none of the other litter mates had gastrointestinal signs and they did not outdoor access. The cat was adopted by a private owner from Turin (Italy) and lived exclusively indoors. Appetite was normal. On clinical examination, the patient had a body condition score of $2 / 5$ and appeared dehydrated (estimated dehydration of 7\%). The level of consciousness was normal. The feces were soft with mucous and streaked with fresh blood. A flotation faecal examination test was negative for roundworms, tapeworms and coccids. A 
prescription diet (Hill's ${ }^{\circledast}$ w/d) and probiotics (Microbiotal ${ }^{\oplus}$; NBF Lanes) were initially prescribed, however, there was no improvement in the clinical signs after three weeks of treatment. Blood work including haematology and biochemistry, serum folate and B12 vitamin were performed and were all within normal limits. ELISA test for feline immunodeficiency virus and feline leukemia virus (Witness FeLV-FIV; Zoetis) were negative. Abdominal ultrasound revealed presence of liquid faeces in the gastrointestinal tract. PCR tests for Tritrichomonas foetus and Cryptosporidium spp. were negative. Flotation faecal examination test was repeated yielding negative results. Cytology on a faecal specimen collected by gentle rectal scraping with a cotton swab revealed the presence of oval to rounded organisms with basophilic internal structure surrounded by a clear capsule (Figure 1, Figure 2). Yeast-sustained diarrhoea was suspected and PCR for Prototheca spp. on faeces was performed. Total genomic DNA was isolated from faecal material by QIAsymphony SP instrument (Qiagen, Milan, Italy), using the DSP Virus/Pathogen Mini kit and following the manufacturer's instructions. PCR was performed in a final reaction volume of $50 \mu \mathrm{L}$, in duplicate, containing $25 \mu \mathrm{L}$ of $2 \times$ HotStarTaq Master Mix (Qiagen, Milan, Italy), 0.3 $\mu \mathrm{M}$ of each primer and $5 \mu \mathrm{L}$ of DNA template; the reaction was brought to the final volume of $50 \mu \mathrm{L}$ with PCR Water. The sequence of the primers N476-F (5'-TCGGAGTTAGCTGGTTCTCC-3') and N476-R

(5'-ATTTTGGGGCCTTAACTGGT-3') to detect all Prototheca spp. was previously described [12], to produce a $216 \mathrm{bp}$ amplicon. The cycling conditions were: initial activation step at $95^{\circ} \mathrm{C}$ for 15 minutes; following by 45 cycles of denaturation at $95^{\circ} \mathrm{C}$ for 30 seconds, annealing at $60^{\circ} \mathrm{C}$ for 30 seconds and extension at $72^{\circ} \mathrm{C}$ for 60 seconds, followed by a final extension for 15 minutes at $72^{\circ} \mathrm{C}$. Negative control, with distilled water, was included in the PCR reactions. The PCR products were separated by capillary gel electrophoresis using the QIAxcel Advanced (Qiagen, Milan, Italy) and represented as electropherograms by the QIAxcel ScreenGel Software, version 1.5 (Qiagen).

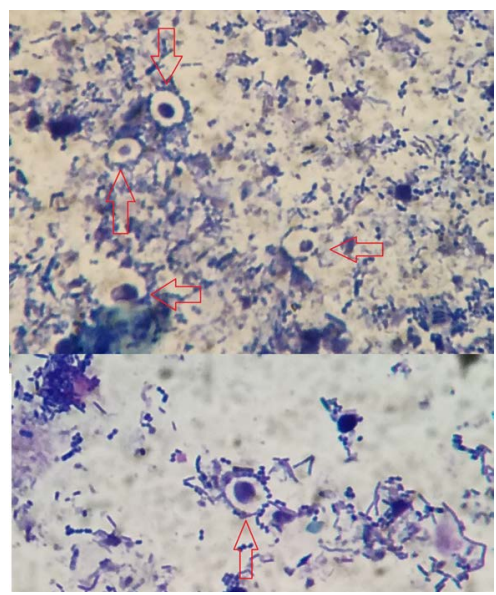

Figure 1. Cytology from a faecal sample collected by rectal scraping revealed the presence (arrowheads) of yeast-like oval to rounded organisms with basophilic internal structure surrounded by clear capsule (Romanowsky staining, 1000× magnification). 


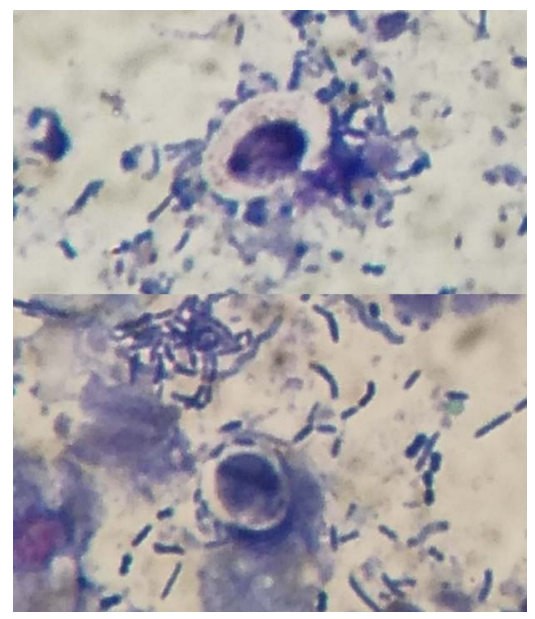

Figure 2. Close up picture of the organisms.

PCR was positive for Prototheca (Figure 3). Itraconazole treatment (Itrafungol $^{\circledR}$; Elanco) at a dose of $5 \mathrm{mg} / \mathrm{kg}$ SID per os was prescribed. The stool quality worsened and the faeces became liquid with undigested food. A hypoallergenic diet (Hill's $\mathrm{s}^{\circledast} \mathrm{z} / \mathrm{d}$ ) and prednisolone (Medrol ${ }^{\circledR} \mathrm{VET}$; Zoetis) at the dose of $0.5 \mathrm{mg} / \mathrm{kg}$ BID per os were added to medical treatment with itraconazole. During the following month the general condition of the cat deteriorated, with reduction in body weight and progressive worsening of the diarrhoea; repeated episodes of rectal prolapse also occurred. Medical management with purse-string sutures were attempted three times. Finally, colopexy was performed and multiple full-thickness intestinal biopsies were obtained. Abdominal lymphadenopathy was observed intraoperatively, however sampling of the abnormal-looking lymph nodes was not performed. Histopathology of duodenum and jejunum intestine showed evidence of chronic mild diffuse neutrophilic and lymphoplasmacellular enteritis and the colon biopsies confirmed chronic mild multifocal lymphoplasmacellular colitis (Figure 4). Prototheca organisms were detected on repeated cytological tests of faecal samples. Treatment with nystatin (Mycostatin $100,000 \mathrm{UI} / \mathrm{mL}$; Sanofi) at the dose of 100,000 UI per os every six hours [13] was introduced, in place of itraconazole, following the owner's consent for the proposed off-label treatment. Several days after introduction of nystatin, clinical signs and faecal characteristics improved. Prednisolone dosage was gradually reduced and discontinued after two weeks. The presence of Prototheca was monitored by performing weekly cytological tests on faecal samples obtained by gentle rectal scraping. After two months of treatment with nystatin 100,000 UI per os every six hours, cytological tests were negative. PCR on faeces was repeated two weeks after the last negative cytological fecal test and it was negative. Nystatin treatment was then interrupted. On follow-up two weeks after the discontinuation of medication, there was no recurrence of the clinical signs. Abdominal ultrasound confirmed resolution of the previously detected lymphadenomegaly (Figure 5). The patient continued to gain weight and was in good body condition at follow-up, one and two years later. Two years after resolution 


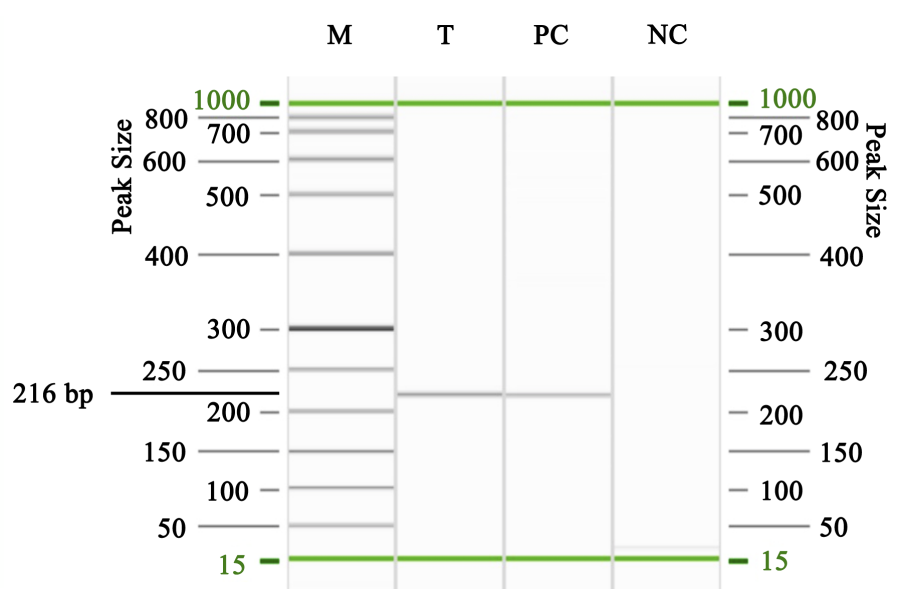

Figure 3. PCR product of the Prototheca strain object of this study, separated by High-resolution capillary electrophoresis. M: QIAxcel DNA Size Marker, 50 - 800 bp Ladder (Qiagen, Milan, Italy). T: DNA template. PC: Positive control. NC: Negative control.

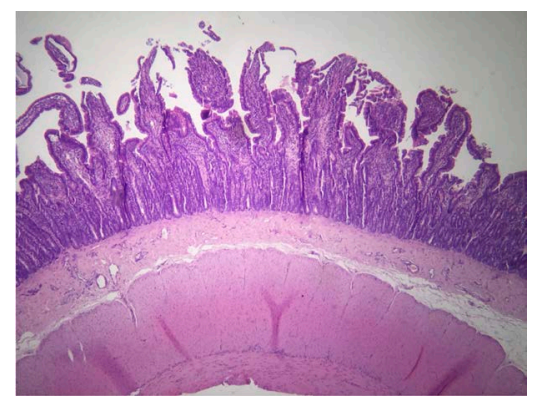

Figure 4. Histopathologic section of jejunum intestine that shows a chronic mild diffuse neutrophilic and lymphoplasmacellular enteritis.

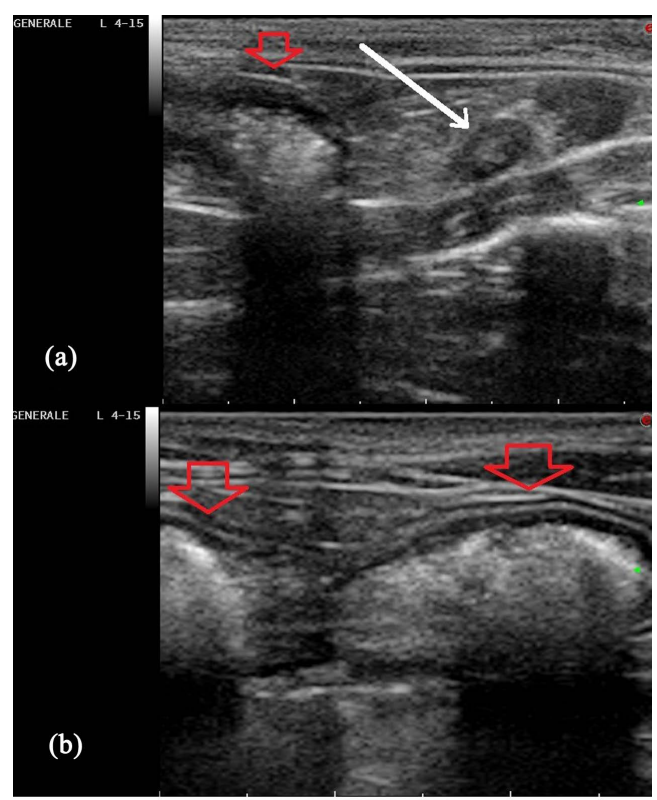

Figure 5. Abdominal ultrasonography (10 MHz linear probe) of the caudal portion of the abdomen. Mild regional lymphadenomegaly (white arrow) of the colon (red arrowhead) at the beginning of nystatin treatment (a) that disappears at the end of the treatment (b). 
of the illness, a new female kitten was introduced in the same domestic environment. At the time of writing the new kitten remained healthy without intestinal symptoms. The owners have never shown anyclinical signs.

\section{Discussion}

Intestinal protothecosis has been described in one human patient [5] and in dogs [9]. In cats, this disease is typically confined to skin nodules and is sensible to local treatment. To the authors' knowledge this is the first case report of intestinal protothecosis in a cat with a positive outcome.

Haemorrhagic colitis is the most common complaint in dogs with protothecosis [7]. Breed predisposition to the disease has recently been suggested for Boxers and Collies, possibly secondary to underlying genetic immunodeficiency [8]. The common progression of the disease features weight loss and progressively worsening chronic diarrhea, with subsequent dissemination of the algae to other sites, with ocular and neurological involvement [8]. Death in dogs is due to generalization of the infection usually within days or weeks [6]. Infection with Prototheca spp. does not cause pathognomonic laboratory and imaging abnormalities [6] [7]. To date, there are no guidelines for the treatment of canine protothecosis [8].

Similar to previous case reports in dogs, intestinal protothecosis caused haemorrhagic colitis with progressive worsening in the general condition in the cat of the present case report. No relevant laboratory abnormalities were detected. The cat was FIV-FeLV negative and no comorbidity was found. In this case there was no generalized dissemination of the infection; this can be explained by the natural resistance of the feline species to infection [9], alongside the beneficial treatment with nystatin. This drug is a polyene antibiotic with antifungal activity, with a spectrum of activity and mechanism of action similar to its novel semisynthetic derivative Amphotericin B and is relatively inexpensive. Nystatin is not absorbed after oral administration and it is almost entirely excreted unchanged in the faeces [14]. Orally administered Nystatin is used primarily for the treatment of oral or gastrointestinal Candida infections in dogs, cats, and birds; it has been used less commonly in other species for the same indications [14]. Nystatin binds to sterols in the membrane of the fungal cell, altering the permeability of the membrane and allowing intracellular potassium and other cellular constituents to "leak out" [14]. In vitro studies have shown that Prototheca spp. is sensitive to Amphotericin $\mathrm{B}$, azoles and a wide range of antibacterial agents [15]. However, none of these compounds have shown convincing efficacy in dogs [7] [16]. When itraconazole was used, alone or together with enrofloxacin, it induced only partial and temporary remission of clinical signs in dogs [8]. In this case report treatment with itraconazole did not improve clinical signs nor reduced the number of Prototheca organisms in repeated fecal cytological exams. On the other hand, nystatin alone appeared to be effective. It is the authors' opinion that treatment with nystatin played a key role in the resolution of the infection sustained by Prototheca in the present case. 
Interestingly, the patient was an exclusively indoor cat. The source of the infection is not known, as none of the other kittens or the owners had symptoms. The potential focus in the domestic environment was not evaluated. The cat's owners did not report any similar symptoms. Protothecosis is not considered a zoonosis, but cases in veterinary patients should be considered as a potential for environmental risk for humans.

The cat is a Bengal cat. Further studies should aim at identifying genetic or breed-predisposing factors in cats, similar to those suspected in Boxer and Collie breeds, as well as the role of the environment in being a favorable condition for infection.

Limitations of the present study are the lack of typing of Prototheca isolated from the intestinal biopsies. Additionally, histopathology of mesenteric lymph nodes was not performed; this would have allowed diagnosis of an inflammatory lymphadenopathy or a potential progression and dissemination of the infectious disease.

This case report suggests that protothecosis should be listed in the differential diagnosis of colitis and diarrhoea in cats. Prototheca infection can be suspected with a simple, inexpensive cytology smears from rectal scrapings. In the authors' opinion nystatin should be considered for the treatment for intestinal protothecosis in cats as well as in dogs. In fact, in dogs the disease appears to be more aggressive than in cats, and no known effective treatment is currently available. Authors declare to have obtained an informed consent from the cat's owners to report this case.

\section{Conflicts of Interest}

The Authors declare that there is no conflict of interest.

\section{Authors' Contributions}

Dr. Sara Manfredini was the clinician responsible for the case management. Dr. Luigi Venco performed cytology on faeces, requested PCR tests for Prototheca and introduced the treatment with nystatin. He also performed abdominal ultrasounds for the case. Dr. Luca Formaggini was responsible for the surgery. Dr. Michele Marino was the consultant for the laboratory PCR test. All four authors contributed to write and edit the manuscript.

The authors thank Prof Laura Kramer, Ph.D., Dip. EVPC, EBVS ${ }^{\mathrm{TM}}$ President, for revising the English language of the manuscript.

\section{References}

[1] Sykes, J.E. (2014) Chapter 70-Protothecosis. In: Sykes, J.E., Eds., Canine and Feline Infectious Diseases, Elsevier, Saunders Inc., St. Louis, USA, 679-685. https://doi.org/10.1016/B978-1-4377-0795-3.00070-3

[2] Danesi, P., Falcaro, C., Binanti, D., et al. (2018) Abstract No: S2.6c. In: Congress Mycology. https://www.isham2018.org/ (Accessed 15 March 2019)

[3] Pfaller, M.A. and Diekema, D.J. (2005) Unusual Fungal and Pseudofungal Infec- 
tions of Humans. Journal of Clinical Microbiology, 43, 1495-1504.

https://doi.org/10.1128/JCM.43.4.1495-1504.2005

[4] Lass-Flor, C. and Mayr, A. (2007) Human Protothecosis. Journal of Clinical Microbiology, 20, 230-242. https://doi.org/10.1128/CMR.00032-06

[5] Konzi, K., et al. (2019) A Case of Intestinal Protothecosis. Médecine et Maladies Infectieuses, 49, 621-623. https://doi.org/10.1016/j.medmal.2019.09.002

[6] Pressler, B.M., Gookin, J.L., Sykes, J.E., et al. (2005) Urinary Tract Manifestations of Protothecosis in Dogs. Journal of Veterinary Internal Medicine, 19, 115-119. https://doi.org/10.1111/j.1939-1676.2005.tb02669.x

[7] Stenner, V.J., Mackay, B., King, T., et al. (2007) Protothecosis in 17 Australian Dogs and a Review of the Canine Literature. Medical Mycology, 45, 249-266. https://doi.org/10.1080/13693780601187158

[8] Bottero, E., Mercuriali, E., Abramo, F., et al. (2016) Fatal Protothecosis in Four Dogs with Large Bowel Disease in Italy. Wiener Tierärztliche Monatsschrift-Veterinary Medicine Austria, Wien.

[9] Nardoni, S. and Mancianti, F. (2018) Micologia in Traversa D. In: Venco, L., Eds., Parassitologia del cane e del gatto, Point Veterinaire Italie, Milano, Italy, 351-376

[10] Sykes, J.E. and Greene, E.G. (2012) Infectious Diseases of the Dog and Cat. 4th Edition, Saunders, Elsevier Inc., St. Louis, USA.

[11] Mahendra, P., Ashebr, A., Tanvir, R., et al. (2014) Protothecosis: An Emerging Algal disease of Humans and Animals. International Journal of Life science and Pharma Research, 3, 16 p.

[12] Capra, E., Cremonesi, P., Cortimiglia, C., Bignoli, G., Ricchi, M., Moroni, P., Pesce, A., Luini, M. and Castiglioni, B. (2014) Simultaneous Identification by Multiplex PCR of Major Prototheca spp. Isolated From Bovine and Buffalo Intramammary Infection and Bulk Tank. Letters in Applied Microbiology, 59, 642-647. https://doi.org/10.1111/lam.12326

[13] Kirk, R.W. (1989) Current Veterinary Therapy X: Small Animal Practice. W.B. Saunders, Philadelphia.

[14] Plumb, D.C. and Pharm, D. (2011) Veterinary Drug Handbook. 7th Edition, PharmaVet Inc., Stockholm, Wisconsin, USA, 1890-1893.

[15] Sapierzynski, R. and Jaworska, O. (2008) Protothecosis as a Cause of Chronic Diarrhoea in a Dog. Polish Journal of Veterinary Sciences, 11, 225-229.

[16] Sobukawa, H., Kano, R., Ito, T., et al. (2011) In vitro Susceptibility of Prototheca zopfii Genotypes 1 and 2. Journal of Medical Mycology, 49, 222-224.

https://doi.org/10.3109/13693786.2010.511285 